\title{
Effect of the Position of Immobilization Upon the Tensile Properties in Injured Achilles Tendon of Rat
}

\author{
Yong Min, $\mathrm{MD}^{1}$, Jeong-Hwan Seo, $\mathrm{MD}^{1}$, Young-Bae Kwon, $\mathrm{MD}^{2}$, Min-Ho Lee, $\mathrm{MD}^{3}$
}

\begin{abstract}
Departments of ${ }^{1}$ Physical Medicine and Rehabilitation and ${ }^{2}$ Pharmacology, Institute for Medical Sciences, Chonbuk National University Medical School and Research Institute of Clinical Medicine, Chonbuk National University Hospital, Jeonju; ${ }^{3}$ Department of Dental Biomaterials, Chonbuk National University School of Dentistry, Jeonju, Korea
\end{abstract}

Objective To examine the effect of the posture of immobilization upon the tensile properties in injured Achilles tendon of rat for an initial period of immobilization.

Methods Forty-two Sprague-Dawley rats were used in the present study. Eighteen rats received a total tenotomy of the right Achilles tendon to mimic total rupture and were divided into three groups comprising of 6 rats each. Ankles of group A were immobilized at $60^{\circ}$ of plantarflexion. Ankles of group B were immobilized at neutral position. Whereas, those of group $\mathrm{C}$ were immobilized at $60^{\circ}$ of dorsiflexion. Other 18 rats received hemitenotomy to mimic partial rupture and were divided into three groups. The remaining 6 rats were kept free as control. After 14 days, we dissected the tendons and analyzed maximum force, stiffness, and energy uptake during pulling of the tendons until they ruptured. The tendons of 6 rats in each group and control were reserved for histology. Picrosirius staining was done for the analysis of collagen organization.

Results In total tenotomy, tensile properties were significantly different between the control and the intervention groups $(p<0.05)$. Group C showed relatively higher values than the groups A and B with respect to tensile properties $(p>0.05)$. In partial tenotomy, tensile properties were significantly different between the control and the intervention groups $(\mathrm{p}<0.05)$. Group $\mathrm{C}$ showed significantly higher value than other intervention groups in terms of maximum force and energy uptake $(\mathrm{p}<0.05)$. The semiquantitative histologic grading scores were assigned for collagen organization. The scores for dorsiflexion posture were higher than the ones for plantarflexion.

Conclusion Dorsiflexion posture in partial ruptured Achilles tendon showed better functional recovery than other immobilized postures. In total ruptured case, the tensile properties showed increasing tendency in dorsiflexion posture.

Keywords Immobilization, Achilles tendon, Biomechanics, Collagen

Received May 3, 2012; Accepted September 5, 2012

Corresponding author: Jeong-Hwan Seo

Department of Physical Medicine and Rehabilitation, Chonbuk National University Medical School, 567 Baekje-daero, Deokjin-gu, Jeonju 561-756, Korea

Tel: +82-63-250-1797, Fax: +82-63-254-4145, E-mail: vivaseo@jbnu.ac.kr

@ This is an open-access article distributed under the terms of the Creative Commons Attribution Non-Commercial License (http://creativecommons.org/ licenses/by-nc/3.0) which permits unrestricted noncommercial use, distribution, and reproduction in any medium, provided the original work is properly cited. Copyright $\odot 2013$ by Korean Academy of Rehabilitation Medicine 


\section{INTRODUCTION}

With the increase in the population of sports activities, the prevalence of tendon injury is on the rise. The Achilles tendon in particular is one of the most frequently affected area [1]. The Achilles tendon receives excessive strain from repeated strenuous activities such as running, jumping, and landing, which can cause structural changes and biomechanical weakening of the tendon, and eventually can lead to rupture [2].

The rehabilitation process begins immediately after injury, thus the therapist is required to have an accurate knowledge of the pathogenesis and of performing adequate and early treatment [3]. In the case of Achilles tendon rupture, both surgical and conservative therapies should be preceded by some immobilization period [4].

The biomechanics of the tendon are determined by microstructural factors such as the content of collagen fibers, fiber orientation, and crosslink density, and the production of collagen fibers, moreover, the collagen fiber array are normally dependent on the direction of tensile force [5-9]. Therefore, the tensile force loaded during the immobilization can change the biochemical constitution and structure of the tendon. Moreover, the tensile force influences the array of collagen fibers, crosslink, extracellular matrix as well as the biomechanical properties of the tendon, and eventually the load and the strength [10-12].

Previous studies have compared the effect of the alteration of tensile forces with different extension and contraction positions of the tendon on the biomechanics of Achilles tendon during the established immobilization period [13-15]. However, the degree of Achilles tendon rupture greatly varies in the clinical setting. Furthermore, there is lack of data on comparison of biomechanical effect of different immobilization methods with severity of rupture or the impact of different immobilization angles combined with differing degrees of tensile forces on the biomechanics of tendon. In this study, we induced acute Achilles tendon injury of different degrees in rats, and compared biomechanical and histological outcomes according to various immobilization angles.

\section{MATERIALS AND METHODS}

\section{Animals}

Forty-two white Sprague-Dawley (SD) rats, aged 8 weeks, weighing 240 to $300 \mathrm{~g}$, were used. They were bred in consistent temperatures of $24^{\circ} \mathrm{C}$ to $26^{\circ} \mathrm{C}$ and humidity up to $60 \%$. The rats were given standard commercial feed.

\section{Intervention and control group assignment}

Of the 42 rats, 36 rats assigned to the intervention group were completely or partially transected their right Achilles tendons in a balanced fashion. The skin of incision site was sutured after the surgery. The 18 rats with complete transection and the other 18 with partial transection were each divided again into three groups of 6 rats according to the immobilization angle: plantarflexion (group A), neutral (group B), and dorsiflexion (group C). The 6 rats in the control group had the skin incision and closure of the hind limb and no other special intervention or immobilization was performed on the Achilles tendon.

\section{Experimental procedure \\ Achilles tendon operation}

The rats were injected with a mixture of $0.1 \mathrm{~mL} / 100 \mathrm{~g}$ of Zoletil (zolazepam+tiletamine) and $0.03 \mathrm{~mL} / 100 \mathrm{~g}$ of Rompun (xylazine). When the rats were fully anesthetized, $1-\mathrm{cm}$ incision was made on the area of the right hind limb to expose the Achilles tendon and depending on the group, complete or partial transection was car-

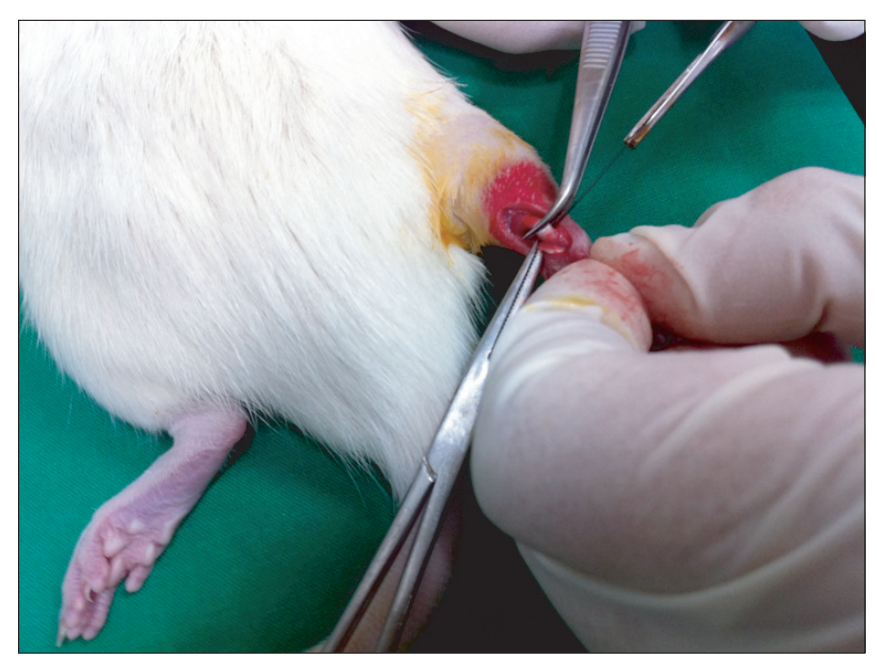

Fig. 1. The figure shows Achilles tendon tenotomy. A $1-\mathrm{cm}$ longitudinal midline incision was made in the skin overlying the Achilles tendon and $1 \mathrm{~cm}$ proximal to the calcaneal insertion, and the tendon was separated from the surrounding tissue. Partial or total tenotomy was done at $1 \mathrm{~cm}$ proximal to the calcaneal insertion. 

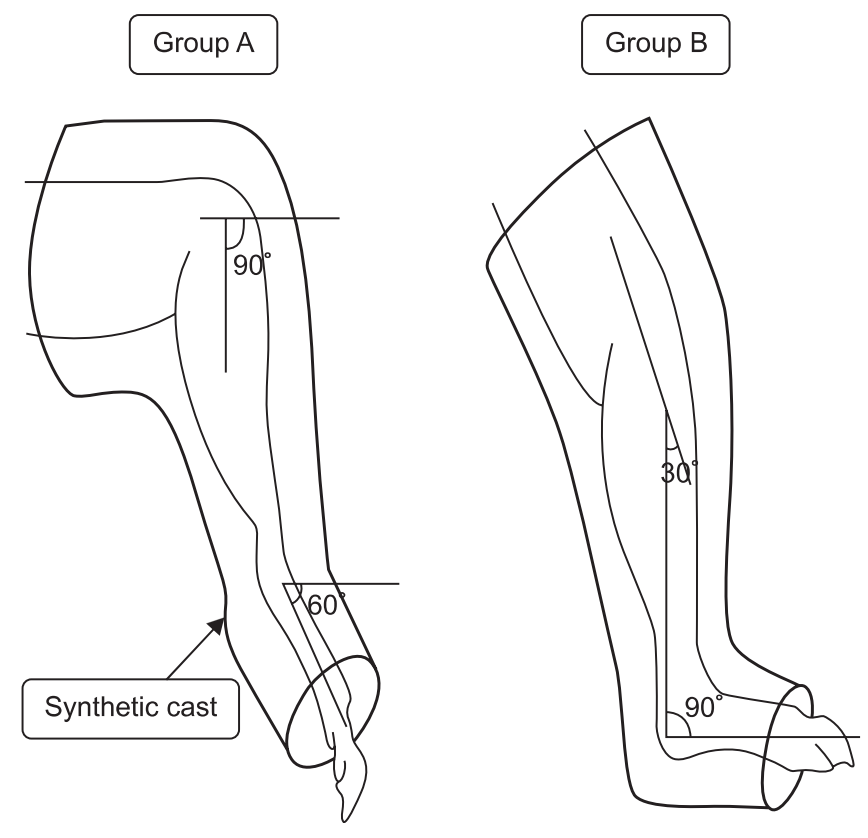

ried out $1 \mathrm{~cm}$ above the attachment of tendon to the calcaneus (Fig. 1) [16]. Subsequently, the incised skin was sutured and $6 \mathrm{mg}$ of gentamycin was intramuscularly injected to prevent postoperative infection.

\section{Hind limb immobilization angle}

After the complete or partial transection of the right hind limb Achilles tendon, thermoplastic was used to immobilize group A at plantarflexion of $60^{\circ}$ of ankle joint. Group B was immobilized with a neutral ankle joint position and group $\mathrm{C}$ was immobilized at dorsiflexion of $60^{\circ}$ of ankle joint (Fig. 2).

\section{Sampling the Achilles tendon}

Two weeks after the immobilization, the 42 animals in the intervention groups and the control group were sacrificed via cervical dislocation, and Achilles tendons were extracted from the affected musculotendinous junction and the attachment site of calcaneus. Each end of the tendon was fixed on a depressor to prevent distortion of the specimen. Five tissue samples from each group were used for the biomechanical test and the remaining one sample was fixed in formalin for the optical microscopic observation.

\section{Biomechanical test}

Thirty tissue samples of Achilles tendons from the intervention groups and 5 from the control group were each installed in Instron-5569 universal testing machine

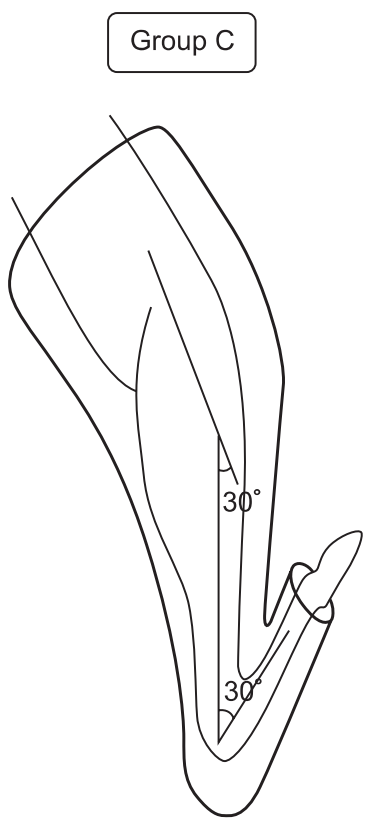

Fig. 2. Schematic diagram of the immobilization. Group A was immobilized at plantarflexion posture of the ankle by $60^{\circ}$ with synthetic cast. Group B was immobilized at neutral posture with cast. Group C was immobilized at dorsiflexion posture of the ankle by $60^{\circ}$.

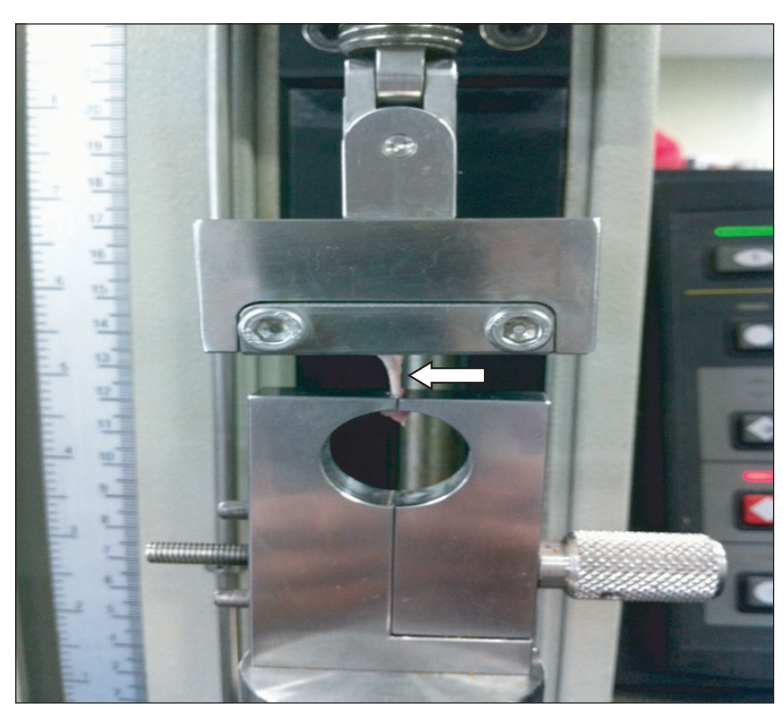

Fig. 3. The figure represents the biomechanical testing machine, Instron. Achilles tendon (arrow) was securely held with clamps and the lesion site was midway between the clamps.

(Instron, Boston, MA, USA) (Fig. 3). The tension test was performed at the speed of $10 \mathrm{~mm} /$ minutes and the start length of the Achilles tendon between the clamping jaws was recorded. The average length of the specimens in the clamping jaws was $9.5 \mathrm{~mm}$. The computer connected to the machine produced the load-extension curve. Maximum force at rupture $(\mathrm{N})$, extension $(\mathrm{mm})$ and maximal elastic force $(\mathrm{N})$ were evaluated from the curve. The stiffness $(\mathrm{N} / \mathrm{mm})$ and the energy absorption to rupture 
(J) were also analyzed (Fig. 4). The stiffness represents the relationship between the change in rate of Achilles tendon against tensile force $(\mathrm{N})$ and the degree of Achilles tendon extension, and the stiffness was calculated as maximal elastic load/extension [13]. The energy absorption at rupture was calculated as the area under the loadextension curve to the point of complete rupture.

\section{Histological assessment}

Two weeks after the surgery, tissue samples of all the groups were fixed in $10 \%$ neutral buffer of formalin. Subsequently, the samples were dehydrated and embedded in paraffin for longitudinal incision at $4-\mu \mathrm{m}$ thickness.

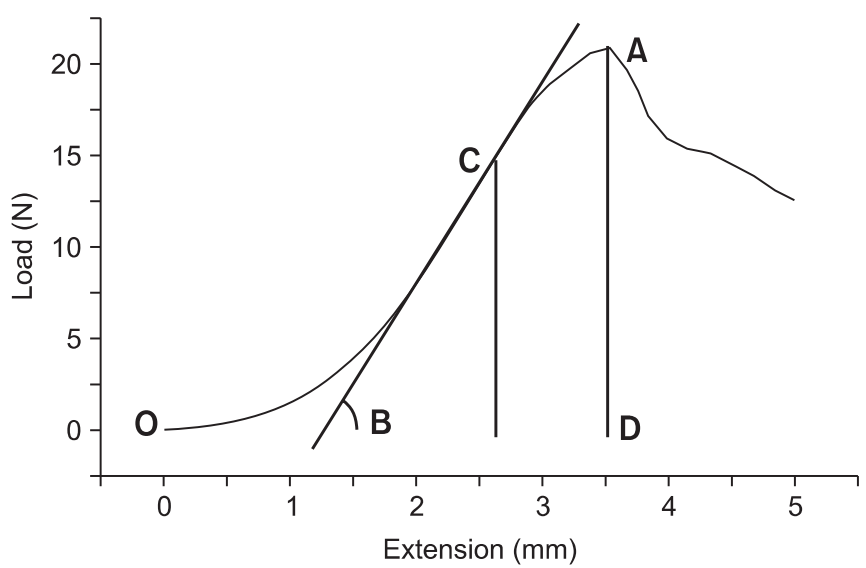

Fig. 4. The results of biomechanical data by Instron. Xaxis represents tendon extension in $\mathrm{mm}$, and $\mathrm{y}$-axis represents load in Newton. Tendon is gradually elongated with tension until rupture. Mark A on the graph signifies the maximum force level the tendon can tolerate, in other words, the maximum force at rupture. Mark B signifies the slope of the graph, which represents the stiffness $(\mathrm{N} /$ $\mathrm{mm}$ ), in other words, it means the elasticity of the tendon. Mark $\mathrm{C}$ signifies the maximum elastic force applied on the tendon when its elasticity is at the highest. The area bordered by $\mathrm{O}, \mathrm{A}$, and $\mathrm{D}$ implies the amount of absorbed energy until the tendon rupture.
The incised tissues were stained with hematoxylin-eosin and the staining intensity of the collagen fibers was analyzed using a standard optical microscope. Picrosirius staining was performed simultaneously to observe the organization of collagen fibers [17]. The staining intensity and the organization of collagen fibers were semiquantitatively stratified into five stages: $1,2,3,4,5$, with 5 being the closest to the normal range [18].

\section{Statistical analysis}

The results of the biomechanical analysis are presented as mean \pm standard deviation. The intervention group was subdivided into complete or partial transection group to compare the changes caused due to the immobilization angle among groups $\mathrm{A}, \mathrm{B}, \mathrm{C}$ and the control using oneway analysis of variance (ANOVA). A post hoc analysis was performed using Bonferroni method comparing the intervention groups with the control as well as among the intervention subgroups. Statistical significance was set as confidence interval of $\mathrm{p}<0.05$. SPSS ver. 18.0 (SPSS Inc., Chicago, IL, USA) was used for statistical analysis.

\section{RESULTS}

\section{Biomechanical outcomes of the complete transection of} Achilles tendon

After complete transaction of the right Achilles tendon, the biomechanical test was performed. The maximum force at rupture in groups $\mathrm{A}, \mathrm{B}$, and $\mathrm{C}$ was $17.30 \pm 1.79$, $18.60 \pm 2.76$, and $21.35 \pm 3.59 \mathrm{~N}$, respectively, and in controls was $29.33 \pm 3.38 \mathrm{~N}$. There was significant difference between all the intervention groups and the control $(\mathrm{p}=0.0001)$. A gradual increase in force at rupture was noted in groups $\mathrm{A}, \mathrm{B}$, and $\mathrm{C}$ in an orderly manner, but there were no significant differences between the intervention subgroups. However, there was significant difference between the control group and each of the interven-

Table 1. Biomechanical parameters at 2 weeks after total tenotomy

\begin{tabular}{lcccrl}
\hline \multicolumn{1}{c}{ Parameter } & Control & Group A & Group B & \multicolumn{1}{c}{ Group C } & p-value \\
\hline Maximum force (N) & $29.33 \pm 3.38$ & $17.30 \pm 1.79^{\mathrm{a})}$ & $18.60 \pm 2.76^{\mathrm{a})}$ & $21.35 \pm 3.59^{\mathrm{a})}$ & $0.0001^{\mathrm{b})}$ \\
\hline Stiffness $(\mathrm{N} / \mathrm{mm})$ & $20.18 \pm 5.61$ & $12.42 \pm 4.65^{\mathrm{a})}$ & $10.19 \pm 2.48^{\mathrm{a}}$ & $8.12 \pm 1.73^{\mathrm{a})}$ & $0.001^{\mathrm{b})}$ \\
\hline Energy (J) & $58.21 \pm 13.69$ & $33.08 \pm 6.30^{\mathrm{a}}$ & $37.02 \pm 16.77$ & $48.97 \pm 14.48$ & $0.0351^{\mathrm{b})}$ \\
\hline
\end{tabular}

Analyzed by one-way analysis of variance (ANOVA) test with Bonferroni post hoc analysis.

${ }^{a)} \mathrm{p}<0.05$, in comparison with the control group. ${ }^{\mathrm{b}} \mathrm{p}<0.05$, in comparison between all the intervention groups and the control. 
tion groups $(\mathrm{p}<0.05)($ Table 1$)$.

The stiffness in groups A, B, and C was 12.42 \pm 4.65 , $10.19 \pm 2.48$, and $8.12 \pm 1.73 \mathrm{~N} / \mathrm{mm}$, respectively, with the control group exhibiting $20.18 \pm 5.61 \mathrm{~N} / \mathrm{mm}$. There were significant differences between all the intervention groups and the control group $(\mathrm{p}=0.001)$. An orderly decrease in the stiffness of Achilles tendon was observed in $\mathrm{A}, \mathrm{B}, \mathrm{C}$ intervention groups, although there was no significant difference between the intervention groups. However, there was significant difference between each of the intervention groups and the control group $(\mathrm{p}<0.05)$ (Table 1).

The energy absorption at rupture in groups A, B, and C was $33.08 \pm 6.30,37.02 \pm 16.77$, and $48.97 \pm 14.48 \mathrm{~J}$, respectively, whereas the control group exhibited $58.21 \pm 13.69$ J. There were significant differences between all the intervention groups and controls $(\mathrm{p}=0.0351)$. The energy absorption at rupture tended to increase in $\mathrm{A}, \mathrm{B}$, and $C$ groups in an orderly fashion; however, there was no significant difference between intervention subgroups. However, group A did show some significant difference when compared to the controls $(\mathrm{p}<0.05)$, whereas groups $B$ and $C$ did not show any significant differences with respect to the control group (Table 1).

\section{Biomechanical outcomes of partial transection of Achilles tendon}

The transection of the right hind limb Achilles tendon was followed by a biomechanical test. The maximum force at rupture in groups $\mathrm{A}, \mathrm{B}$, and $\mathrm{C}$ was $17.61 \pm 5.71$, $18.67 \pm 3.00$, and $26.03 \pm 2.58 \mathrm{~N}$, respectively, with $29.33 \pm$ $3.38 \mathrm{~N}$ in the control group. There were significant differences between all the intervention groups and the controls $(\mathrm{p}=0.0004)$. In a subgroup comparison, the force at rupture increased in A, B, and C groups in an order and there were significant differences between group $C$ and groups $A$ and $B(p<0.05)$. In addition, groups $A$ and $B$ showed lower maximum force at rupture when compared to the control group $(\mathrm{p}<0.05)$, whereas group $\mathrm{C}$ did not show any difference $(\mathrm{p}<0.05)$ (Table 2$)$.

The stiffness in groups A, B, and C was $14.71 \pm 3.82$, $13.63 \pm 8.94$, and $10.03 \pm 5.22 \mathrm{~N} / \mathrm{mm}$, respectively, and that in the control group was $20.18 \pm 5.61 \mathrm{~N} / \mathrm{mm}$. There were no significant differences between all the intervention groups and the controls $(\mathrm{p}=0.1157)$. The Achilles tendon stiffness tended to make a steady decline in A, B, and C groups in an orderly manner, and there were no statistical significance among intervention subgroups as well as between the intervention groups and the control group (Table 2).

The energy absorption at rupture in groups A, B, and $\mathrm{C}$ was $33.96 \pm 4.01,39.07 \pm 7.86$, and $57.22 \pm 14.67 \mathrm{~J}$, respectively, and that in the control group was $58.21 \pm 13.69 \mathrm{~J}$. There were statistically significant differences between all of intervention groups and the controls $(p=0.0046)$. The energy absorption at rupture was the highest in group $\mathrm{C}$ amongst the intervention subgroups, and there was a significant difference between group C and group A ( $\mathrm{p}<0.05)$, but not with group B. Group A was the only intervention group, which exhibited significant difference when compared to the controls $(\mathrm{p}<0.05)$ (Table 2).

\section{Histological outcomes}

Histological outcomes are provided as supplementary data. The Achilles tendons of 36 rats in the intervention groups were smoothly reconnected by naked eyes two weeks after the operation. In the hematoxylin-eosin staining, both the total transection (Fig. 5) and partial transection (Fig. 6) groups were immobilized in varying positions. When the outcomes of the plantarflexion and the dorsiflexion positions were compared semiquantitatively, the intensity of staining of collagen fibers was 4 without any difference. In a semiquantitative comparison of the collagen fibers organization as per Picrosirius

Table 2. Biomechanical parameters at 2 weeks after hemitenotomy

\begin{tabular}{llllll}
\hline \multicolumn{1}{c}{ Parameter } & Control & Group A & Group B & Group C & p-value \\
\hline Maximum force (N) & $29.33 \pm 3.38$ & $17.61 \pm 5.71^{\mathrm{a}, \mathrm{b})}$ & $18.67 \pm 3.00^{\mathrm{a}, \mathrm{b})}$ & $26.03 \pm 2.58$ & $0.0004^{\mathrm{c})}$ \\
\hline Stiffness (N/mm) & $20.18 \pm 5.61$ & $14.71 \pm 3.82$ & $13.63 \pm 8.94$ & $10.03 \pm 5.22$ & 0.1157 \\
\hline Energy (J) & $58.21 \pm 13.69$ & $33.96 \pm 4.01^{\mathrm{a}, \mathrm{b})}$ & $39.07 \pm 7.86^{\mathrm{a})}$ & $57.22 \pm 14.67$ & $0.0046^{\mathrm{c})}$ \\
\hline
\end{tabular}

Analyzed by one-way analysis of variance (ANOVA) test with Bonferroni post hoc analysis.

${ }^{a)} \mathrm{p}<0.05$, in comparison with the control group. ${ }^{\text {b }} \mathrm{p}<0.05$, in comparison with group $\mathrm{C}$. ${ }^{\text {c) }} \mathrm{p}<0.05$, in comparison between all the intervention groups and the control. 


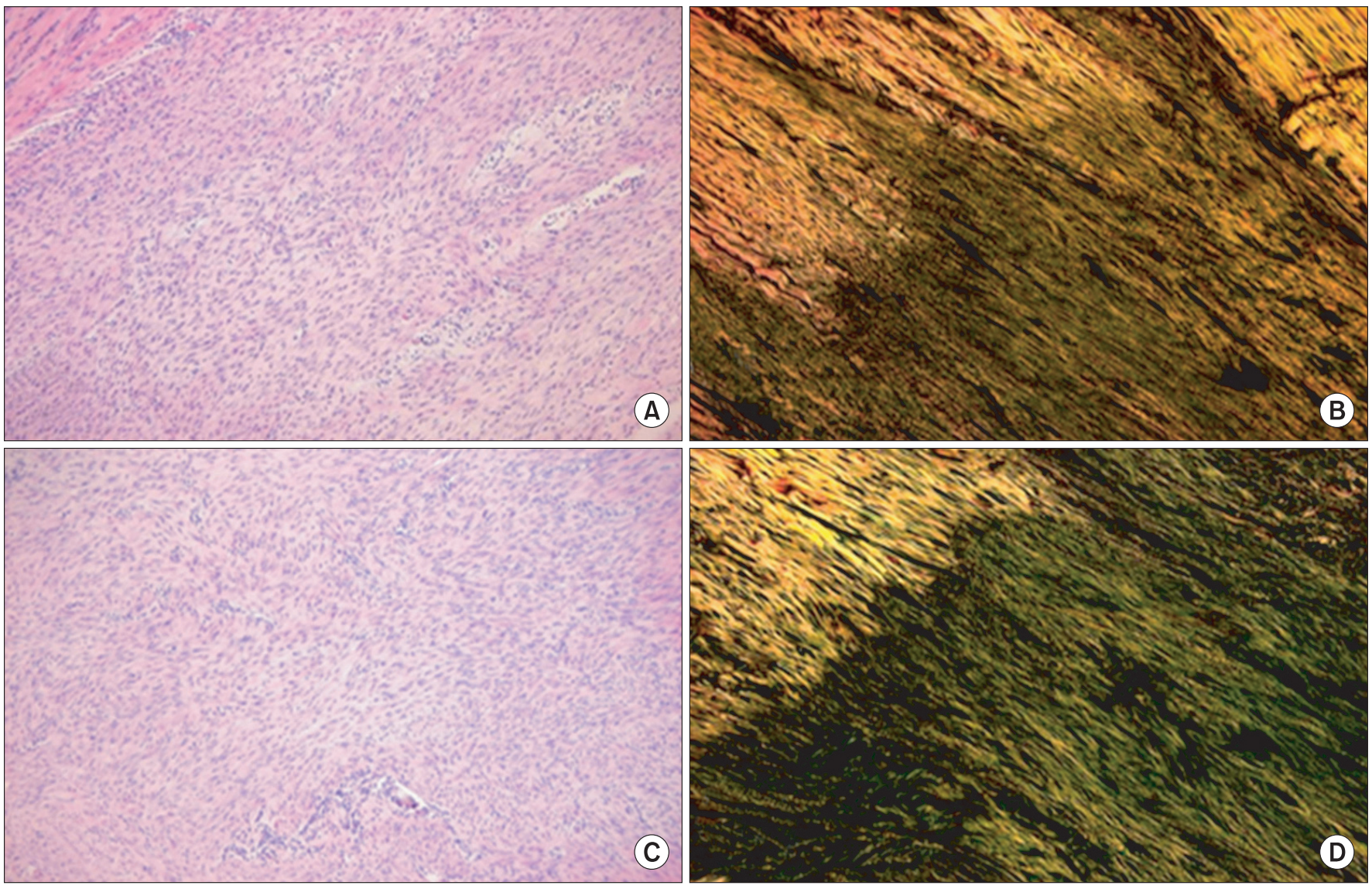

Fig. 5. $(A, C)$ are hematoxylin-eosin $(\times 100)$ staining and $(C, D)$ are the Picrosirius $(\times 100)$ staining of longitudinal sections through the rupture site of the Achilles tendon in total tenotomy. Among the total tenotomy rat tendons, (A, B) are from the plantarflexion and (C, D) are from the dorsiflexion posture of immobilization. Green fibers indicate type III collagen in (B, D). In (B, D), the alignment of tendon fibers can be seen. However, (D) of dorsiflexion posture shows denser and well aligned collagen fibers than (B).

staining, the total transection group with plantarflexion showed a score of 1 , and the dorsiflexion group showed a score of 2 (Fig. 5). The partial transection group with plantarflexion had a score of 1 , whereas the dorsiflexion group exhibited a score of 3 . According to the staining results, the dorsiflexion group exhibited improved organization of collagen fibers among all the intervention subgroups (Fig. 6).

\section{DISCUSSION}

This study provided differing degrees of distraction force with various degrees of Achilles tendon injury and ankle joint immobilization angles, which aided in the identification of the biomechanical effect of load upon the recovery of Achilles tendon, and ultimately in assessing the impact of immobilization angle on the recovery of the Achilles tendon. In the biomechanical test, the force at rupture and the energy absorption at rupture of healed Achilles tendons increased in the complete rupture group with dorsiflexion and almost reached the level of the control group of intact Achilles tendons. In the partial transection group, dorsiflexion group withstood the significantly highest force at rupture amongst other immobilization positions. Furthermore, the energy absorption at rupture was also higher with dorsiflexion when compared to plantarflexion. The supplementary histological data revealed improvement in histological organization of collagen fibers with dorsiflexion position than plantarflexion in both complete and partial transection groups. This result indicates that dorsiflexion at immobilization induces more distraction force that accelerates the healing process.

Murrell et al. $[19,20]$ and Best et al. [21] have reported 

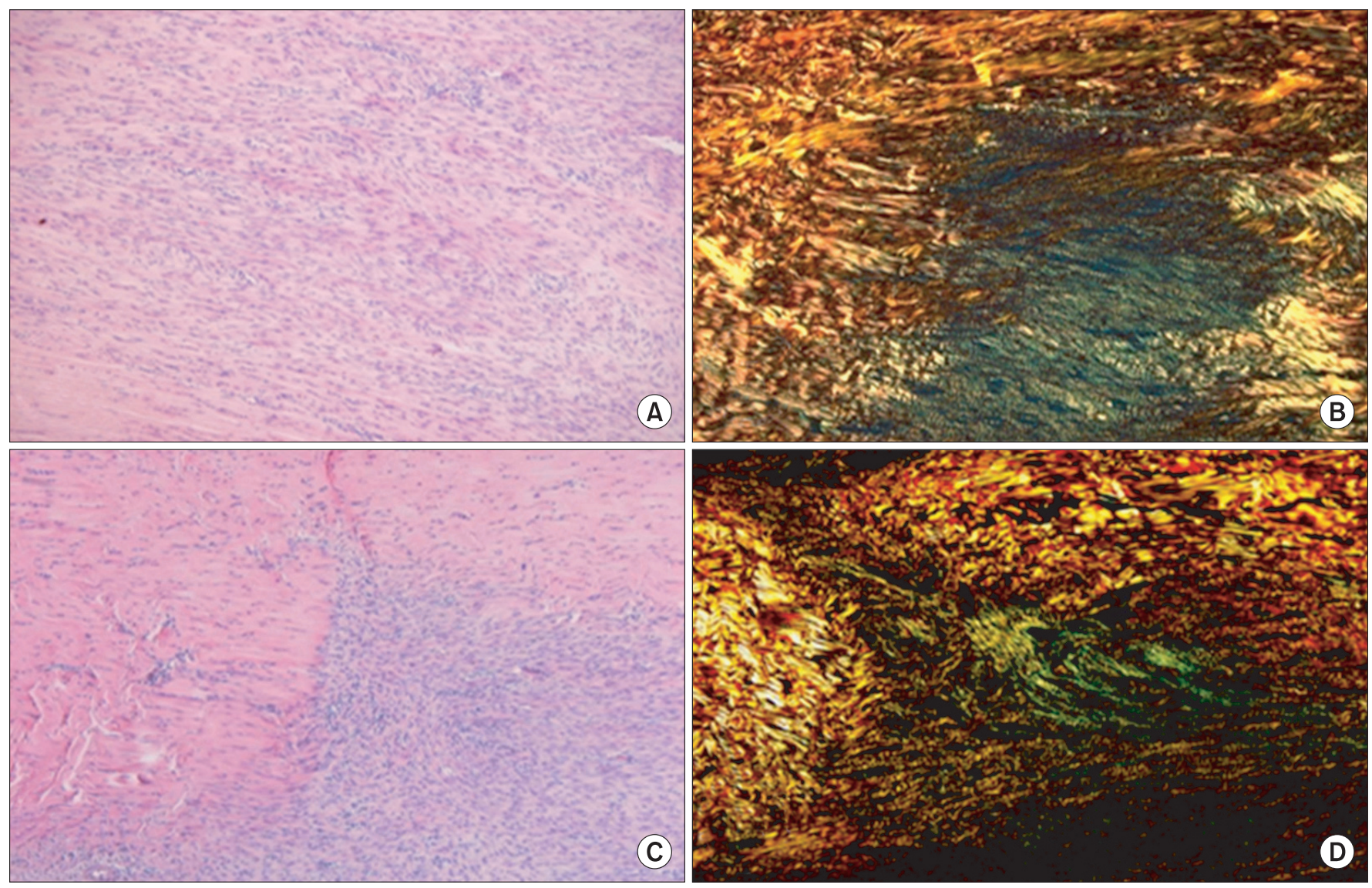

Fig. 6. $(A, C)$ are hematoxylin-eosin $(\times 100)$ staining and $(B, D)$ are the Picrosirius $(\times 100)$ staining of longitudinal sections through the rupture site of the Achilles tendon in hemitenotomy. (A, B) are from the plantarflexion and (C, D) are from the dorsiflexion posture of immobilization. Green fibers indicate type III collagen. In hemitenotomy, (D) from dorsiflexion posture also shows denser collagen fibers than (B).

that the regeneration of the Achilles tendon takes about 15 days in rat models. Several studies have suggested that the immobilization clinically causes degeneration and changes in the characteristics of musculoskeletal tissue $[5,11,22]$. Also, it has been reported that when elastic stress is induced onto the recovering tendon in order to reduce degeneration during the early immobilization after the Achilles tendon injury, the increasing migration of fibroblasts result in significant decrease in the failure of early postoperative treatment [19]. Bidder et al. [23] have reported that biomechanical load increases the synthesis of vascular endothelial growth factor to mediate angiogenesis. However, in a qualitative histologic comparison, the area of injury area in our study showed no difference in terms of extension and constitution of the granulation tissue, and the distribution of lymphocyte and fibroblast within the intervention groups.

Mass et al. [24] have hypothesized that the biomechani- cal load on the healing area might contribute to the organic array of deposited collagen fibers and strengthening of extracellular matrix bonding to reinforce the regenerated tissue. These fibers are arrayed parallel to the direction of induced force, which enhances them to withstand stronger tensions. The exposure to load during the postoperative rehabilitation period boosts the resistance of tissues against deformation [24]. This report is consistent with the finding in the current study suggesting that the immobilization angle of increasing load leads to better biomechanical outcomes.

The Picrosirius staining revealed that the dorsiflexion group (group C) had more clearly organized and thickened collagen fibers. This observation strongly suggests that the biomechanical load on the treated area contributes to more organic array of collagen fibers and improvement in healing process. This histological difference explains as why the group $\mathrm{C}$ exhibited highest bio- 
mechanical test results.

The recovery of stiffness is associated with the proliferation of collagen fibers as well as a number of other factors such as the maximum force at load or rupture related to the tension [25]. This study demonstrated a trend towards decrease in stiffness of tendon with dorsiflexion during the immobilization in both the transection groups. Several animal studies reported that the force at rupture returns to normal on 13th day after injury, but only $60 \%$ with respect to stiffness, and the tendon stiffness might be reduced regardless of the improvement in the rupture load and histological outcomes $[11,21,26]$. If the biomechanical test had been carried out at later stage, the results might have shown similar degree of stiffness recovery as the force at rupture and energy absorption at rupture.

This study revealed that dorsiflexion was more beneficial within the first two weeks of healing after the injury. However, the observation period was too short to examine subsequent changes in biomechanical properties and array of collagen fibers. Another limitation of this study is the small number of animals used in the biomechanical assessment. Further studies are needed to determine more effective immobilization period, selection of intervention group, and the new treatment options.

In conclusion, this study compared the biomechanical and histological outcomes of different immobilization methods for various degrees of Achilles tendon injury. In the partial transection group, dorsiflexion resulted in improved biomechanical performance and histological findings when compared to plantarflexion. In the complete transection group, biomechanical performance also seemed to improve with dorsiflexion. This might reflect the improvement in tensile strength of tendon due to more organic array of collagen fibers through consistent and repeated biomechanical strain on the affected area. It is hypothesized that this study will provide a rationale for future clinical trials on the immobilization methods of the injured Achilles tendon.

\section{CONFLICT OF INTEREST}

No potential conflict of interest relevant to this article was reported.

\section{REFERENCES}

1. Thompson J, Baravarian B. Acute and chronic Achilles tendon ruptures in athletes. Clin Podiatr Med Surg 2011;28:117-35.

2. Matsumoto F, Trudel G, Uhthoff HK, Backman DS. Mechanical effects of immobilization on the Achilles' tendon. Arch Phys Med Rehabil 2003;84:662-7.

3. Lee JH, Kim HS, Ahn KH. Effects of local corticosteroid injection and weight bearing on injured Achilles tendon in a rat model. J Korean Acad Rehabil Med 2002;26:215-22.

4. Longo UG, Ronga M, Maffulli N. Acute ruptures of the achilles tendon. Sports Med Arthrosc 2009;17:127-38.

5. Amiel D, Woo SL, Harwood FL, Akeson WH. The effect of immobilization on collagen turnover in connective tissue: a biochemical-biomechanical correlation. Acta Orthop Scand 1982;53:325-32.

6. Harwood FL, Amiel D. Differential metabolic responses of periarticular ligaments and tendon to joint immobilization. J Appl Physiol 1992;72:1687-91.

7. Savolainen J, Myllyla V, Myllyla R, Vihko V, Vaananen $\mathrm{K}$, Takala TE. Effects of denervation and immobilization on collagen synthesis in rat skeletal muscle and tendon. Am J Physiol 1988;254(6 Pt 2):R897-902.

8. Walsh S, Frank C, Hart D. Immobilization alters cell metabolism in an immature ligament. Clin Orthop Relat Res 1992;(277):277-88.

9. Woo SL, Gomez MA, Sites TJ, Newton PO, Orlando CA, Akeson WH. The biomechanical and morphological changes in the medial collateral ligament of the rabbit after immobilization and remobilization. J Bone Joint Surg Am 1987;69:1200-11.

10. Almeida-Silveira MI, Lambertz D, Perot C, Goubel F. Changes in stiffness induced by hindlimb suspension in rat Achilles tendon. Eur J Appl Physiol 2000;81:2527.

11. Loitz BJ, Zernicke RF, Vailas AC, Kody MH, Meals RA. Effects of short-term immobilization versus continuous passive motion on the biomechanical and biochemical properties of the rabbit tendon. Clin Orthop Relat Res 1989;(244):265-71.

12. Mullner T, Kwasny O, Reihsner R, Lohnert V, Schabus R. Mechanical properties of a rat patellar tendon stress-shielded in situ. Arch Orthop Trauma Surg 2000;120:70-4. 
13. Trebacz H. Effect of immobilization in a lengthened position on mechanical properties of the Achilles tendon in growing rats. Acta Bioeng Biomech 2005;7:7986.

14. Herbert RD, Balnave RJ. The effect of position of immobilisation on resting length, resting stiffness, and weight of the soleus muscle of the rabbit. J Orthop Res 1993;11:358-66.

15. Kim KC, Hwang DS, Chung SY, Woo SM, Lee CH. Biomechanical and histological effects of different regimens of immobilzation after operative treatment in a ruptured rabbit Achilles tendon. J Korean Orthop Assoc 2005;40:340-6.

16. Dogan A, Korkmaz M, Cengiz N, Kalender AM, Gokalp MA. Biomechanical comparison of Achilles tenotomy and achilloplasty techniques in young rats: an experimental study. J Am Podiatr Med Assoc 2009;99:216-22.

17. Schizas N, Li J, Andersson T, Fahlgren A, Aspenberg P, Ahmed M, et al. Compression therapy promotes proliferative repair during rat Achilles tendon immobilization. J Orthop Res 2010;28:852-8.

18. Glazebrook MA, Wright JR Jr, Langman M, Stanish WD, Lee JM. Histological analysis of achilles tendons in an overuse rat model. J Orthop Res 2008;26:840-6.

19. Murrell GA, Lilly EG 3rd, Collins A, Seaber AV, Goldner RD, Best TM. Achilles tendon injuries: a comparison of surgical repair versus no repair in a rat model.
Foot Ankle 1993;14:400-6.

20. Murrell GA, Lilly EG 3rd, Goldner RD, Seaber AV, Best TM. Effects of immobilization on Achilles tendon healing in a rat model. J Orthop Res 1994;12:582-91.

21. Best TM, Collins A, Lilly EG, Seaber AV, Goldner R, Murrell GA. Achilles tendon healing: a correlation between functional and mechanical performance in the rat. J Orthop Res 1993;11:897-906.

22. Larsen NP, Forwood MR, Parker AW. Immobilization and retraining of cruciate ligaments in the rat. Acta Orthop Scand 1987;58:260-4.

23. Bidder M, Towler DA, Gelberman RH, Boyer MI. Expression of mRNA for vascular endothelial growth factor at the repair site of healing canine flexor tendon. J Orthop Res 2000;18:247-52.

24. Mass DP, Tuel RJ, Labarbera M, Greenwald DP. Effects of constant mechanical tension on the healing of rabbit flexor tendons. Clin Orthop Relat Res 1993;(296):301-6.

25. Liu SH, Yang RS, al-Shaikh R, Lane JM. Collagen in tendon, ligament, and bone healing: a current review. Clin Orthop Relat Res 1995;(318):265-78.

26. Noyes FR. Functional properties of knee ligaments and alterations induced by immobilization: a correlative biomechanical and histological study in primates. Clin Orthop Relat Res 1977;(123):210-42. 